\title{
Matériaux pour l'étude de la théorie de la narration dans la rhétorique humaniste
}

Jean Lecointe

\section{(2) OpenEdition}

1 Journals

Édition électronique

URL : https://journals.openedition.org/rhetorique/1316

DOI : $10.4000 /$ rhetorique. 1316

ISSN : 2270-6909

Éditeur

UGA Éditions/Université Grenoble Alpes

Édition imprimée

ISBN : 978-2-37747-345-8

Référence électronique

Jean Lecointe, " Matériaux pour l'étude de la théorie de la narration dans la rhétorique humaniste », Exercices de rhétorique [En ligne], 18 | 2022, mis en ligne le 24 février 2022, consulté le 26 février 2022. URL : http://journals.openedition.org/rhetorique/1316; DOI : https://doi.org/10.4000/rhetorique.1316

Ce document a été généré automatiquement le 26 février 2022.

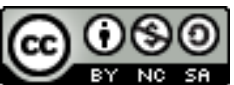

Les contenus de la revue Exercices de rhétorique sont mis à disposition selon les termes de la Licence Creative Commons Attribution - Pas d'Utilisation Commerciale - Partage dans les Mêmes Conditions 4.0 International. 


\title{
Matériaux pour l'étude de la théorie de la narration dans la rhétorique humaniste
}

\author{
Jean Lecointe
}

Les matériaux proposés ici rassemblent, de façon cursive, des éléments tirés d'un certain nombre de traités (et, en annexe, de quelques commentaires de traités). L'inventaire ne prétend pas à l'exhaustivité. Le but est que le panorama ainsi obtenu donne les principaux traits de la réflexion rhétorique humaniste sur la narration. Le latin uirtutes (avec majuscule, Virtutes) signifiera partout « qualités ", ou, en langue ancienne, « vertus » de la narration, par opposition à uitia, « vices » c'est-à-dire « défauts».

On trouvera en ligne la plupart des références anciennes mentionnées. Les chiffres romains désignent uniquement la subdivision en livres de l'ouvrage cité ; les chiffres arabes, la subdivision en chapitres ou, selon les cas, en paragraphes. Quand il ne s'agit pas de la première édition, nous indiquons la date de celle-ci entre parenthèses, juste après le titre. Pour les dates de princeps et les graphies des noms (auteurs et éditeurs), en général nous nous fions à Renaissance rhetoric ${ }^{1}$, avec un œil sur les « notices d'autorité » de la BnF (data.bnf.fr). Pour la liste des rééditions d'un même ouvrage, souvent très nombreuses, voir de même Renaissance rhetoric. Enfin, nous signalons chaque fois les pages où Peter Mack donne une description des auteurs ou ouvrages que nous mentionnons (dans $A$ history of Renaissance rhetoric 1380-1620, Oxford, Oxford University Press, 2011).

Abréviations : « Her. », Rhétorique à Herennius ; « De or. », « Inv. » et «Part. » renvoient aux traités de Cicéron, respectivement De l'orateur, De l'invention et Divisions de l'art oratoire (en latin, Partitiones oratoriae). 


\section{1. «Trois modes »}

Platon, République, III, 392d-398b

Oppose $(\dot{\alpha} \pi \lambda \dot{\eta}) \delta i \eta \gamma \dot{\eta} \sigma \iota, \mu \imath \mu \dot{\eta} \sigma \varsigma \varsigma$ et énonciation mixte (terminologie imprécise) : pour «le récit raconté d'événements ou passés, ou présents, ou futurs", les "conteurs d'histoires et les poètes" ont recours "soit à un récit simple [haplè diégèsis], soit à un récit issu d'une imitation [mimèsis], soit encore à une forme mixte » (392d, trad. G. Leroux, dans Platon, CEuvres complètes, dir. L. Brisson, Paris, Flammarion, 2011, p. 1553-1554; suivent les exemples).

\section{Aristote, Poétique, 3, 1448a}

Distinction de base, en termes confus.

3 Diomède, Ars grammatica, Paris, Jean Petit, 1507, h iiii vo

Texte de référence, repris sans cesse, avec les terminologies grecque et latine: "Poematis genera sunt tria. aut enim actiuum est uel imitatiuum quod graeci $\Delta \rho \alpha \mu \alpha \tau \imath k o v$

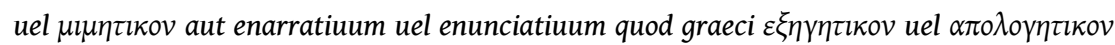
dicunt ", " Il y a trois genres de poème ; soit actif ou imitatif, que les Grecs appellent Dramatikon ou mimètikon, soit narratif ou énonciatif que les Grecs nomment exègètikon ou apologètikon". Diomède ajoute le genre "commune uel mixtum quod

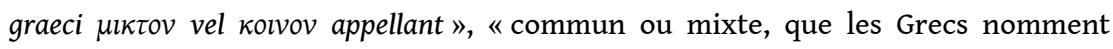
mikton ou koinon".

4 Giovanni Francesco Conti Stoa (Quintianus Stoa, 1484-1557), Epographiae, Pavie, J. P. de Burgofranco, 1511, I, 6 « De poeticae artis speciebus », fo $2 \mathrm{v}^{\circ}$

Doctrine canonique d'après Diomède, lu dans un autre texte, ou corrigé. Terminologie grecque et latine: "actiuam siue imitatiuam a graecis $\delta \rho \alpha \mu \alpha \tau \kappa \eta \nu$ uel $\mu \imath \mu \eta \tau \imath \kappa \eta v$ dictam [...]. Enarratiuam siue enunciatiuam: quae graecorum $\varepsilon \xi \eta \gamma \varepsilon \mu \alpha \tau \imath \kappa \eta$ vel

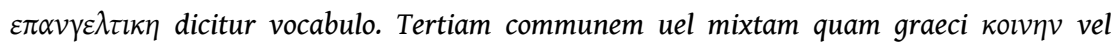
$\mu l \kappa \tau \eta v$ nominant ", " active ou imitative, dite par les Grecs dramatikê ou mimêtikê [...]. Énarrative ou énonciative, en grec exègèmatikè ou epangeltikè. La troisième commune ou mixte, en grec koinè ou miktè ». Les chapitres suivants subdivisent les trois espèces.

5 François Dubois (Franciscus Silvius ou Sylvius, mort vers 1530), Francisci Syluii Ambianatis Poetica (1516), Paris, Josse Bade, 1520, a iiii $\mathrm{r}^{\circ}$

Distingue trois genres ou genera de la narratio, d'abord: historicum, oratorium, poeticum, « historique, oratoire, poétique », puis subdivise le poeticum en trois autres genera, en calquant probablement Quintianus Stoa.

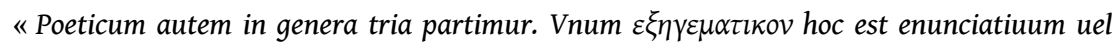
narratiuum in quo poeta omnia sine ulla personarum interlocutione narrat. Vt Georgica Virgilii. De quo itidem, propterea quod ad oratorium uel historicum accedit propius, nihil dicendum putauimus. Alterum $\delta \rho \alpha \mu \alpha \tau \kappa o v$, hoc est repraesentatiuum uel actuosum, in quo personae solae absque ulla poetae authorisue interlocutione agunt, ut comoediae, ut

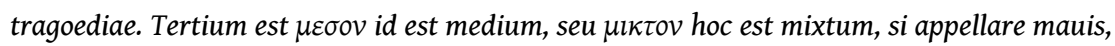
quod scilicet ex utroque constat." "On divisera le poétique en trois genres. L'un, exègèmatikon, c'est-à-dire énonciatif ou narratif, où le poète s'exprime toujours sans faire intervenir de personnages ; ainsi les Géorgiques de Virgile. Ce n'est pas la peine d'en parler ici, puisque cela se rapproche de l'oratoire ou de l'historique. Un second, dramatikon, c'est-à-dire représentatif et en acte, où les personnages s'expriment sans intervention du poète ou de l'auteur. Un troisième enfin, meson, c'est-à-dire moyen, ou mikton, c'est-à-dire mixte, si l'on préfère, autrement dit composé des deux autres. »

6 François Pomey (1618-1673), Candidatus rhetoricae (1659), Munich, Johann Wilhelm Schell, 1664, p. 289 
Comme espèces de la narratio poetica ou "narration poétique »; actiuum, enunciatiuum et mixtum (" actif, énonciatif et mixte»); et terminologie grecque de Diomède.

\section{La narratio comme exercice préparatoire}

Aelius Théon, Progymnasmata, primae apud rhetorem exercitationes, éd. et trad. latine de Joachim Camerarius, Bâle, Johann Oporinus, 1541, p. 253 ; Progymnasmata, éd. et trad. Michel Patillon, Paris, Belles Lettres, 1997

Extrêmement détaillé, sur tous les exercices, notamment les trois premiers, la chrie (Patillon p. 18-30=Spengel 96-106), la fable (muthos, p. 253-263 de l'éd.1541; Patillon p. 30-38 $=72-78$ ) et le récit (diègèma, p. 264-288 de l'éd. 1541 ; Patillon p. 38-61 =78-96) ; est le traité le plus complet de la narration laissé par l'Antiquité, avec analyses littéraires (Homère, etc.); analyse en particulier l'oeconomia ou organisation narrative de l'Odyssée (p. 278, Patillon p. 48-49 = 86, nous citons infra le début du passage); trois uirtutes (p. 266, Patillon p. $40=79.20$ ); lieux; vices; vocabulaire; ordre; figures. Le plus ancien et le plus riche des manuels de "préexercices » ou "exercices préparatoires", en grec progymnasmata, en latin praeexercitamenta. Mais peu connu avant le milieu du XVI ${ }^{\mathrm{e}}$ siècle.

8 Aphthonios (Aphthonius ou Aphtonius), Progymnasmata, I et II, éd. et trad. Michel Patillon, dans Corpus rhetoricum, Paris, Belles Lettres, 2008

Proche d'Hermogène, auquel il s'est souvent substitué dans le corpus hermogénien au cours du Moyen Âge; plus succinct. La fable et le récit (fabula, narratio, Patillon p. 112-114) sont les deux premiers exercices, comme chez Hermogène. Propose des divisions, des consignes et des exemples. Divisions, en particulier de la narratio : en

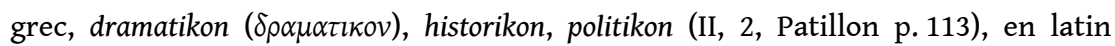
poetica, historica, ciuilis (" poétique, historique, politique», grande fortune ultérieure de cette division ${ }^{2}$ ). Consignes : proches des consignes usuelles; parmi celles-ci, quatre uirtutes : perspicuitas, breuitas, probabilitas, uerborum proprietas (dans la trad. d'Aphthonius par Agricola), soit «clarté, concision, fiabilité, justesse de l'expression » (« correction de la langue », selon la trad. Patillon, le grec étant « tôn onomatôn hellènismos », II, 4, p. 114). Pour la « chrie », voir infra.

De cet ouvrage extrêmement répandu, il faut surtout signaler l'édition commentée de Reinhard Lorich (Lorichius), qui fusionne les deux traductions latines de Cattaneo et d'Agricola, en ajoutant une abondante annotation : Aphthonii Sophistae Progymnasmata partim a Rodolpho Agricola partim a Iohanne Maria Catanaeo latinitate donata [...] cum [...] Scholiis Rheinhardi Lorichii... (1542), Cologne, Birckmann, 1562, $\mathrm{f}^{\circ}$ 1-20 $\mathrm{r}^{\mathrm{o}}$. Ces pages donnent un véritable petit traité de la fable et de la narration, avec de nombreux exemples, classiques ou inventés par le commentateur Lorich. Traite abondamment la question des narrations poétiques. Riches exemples (Ovide, etc.). En général reprend les données canoniques, avec une attention particulière pour les lieux, toutefois. Trois modes selon Diomède.

9 Pseudo-Hermogène, Exercices préparatoires, 1 et 2, éd. et trad. Michel Patillon, dans Corpus rhetoricum, Paris, Belles Lettres, 2008, p. 180-185

Mentionne «fable » et « récit » comme premiers exercices scolaires - à noter que le troisième exercice, la « chrie » (voir Bibliographie infra, Ch. Noille), peut prendre un aspect narratif, s'il s'agit d'une «chrie active» ou «chrie d'acte». Mais les consignes sont très sommaires. La traduction latine, très répandue au Moyen Âge, est celle de Priscien: Hermogenis Rhetoris ad artem oratoriam Praeexercitamenta, Prisciano interprete, Nuremberg, Johann Petreius, 1544.

Quintilien, Institution oratoire, II, 4 « Des premiers exercices chez le rhéteur », § 2-5 ; éd. et trad. Jean Cousin, Paris, Belles Lettres, t. II, 1976, p. 36-37 
Distingue, à ce propos, fabula, argumentum et historia. Mentionne aussi les narrations poeticas, qu'il réserve au grammaticus. Voir aussi, notamment sur ce dernier point I, 9.

\section{(1556), Barcelone, S. a Cormellas, 1593, I, p. 5, « De progymnasmatis»}

Très détaillé, sur les trois premiers exercices; exemples. Établit une correspondance entre ces exercices et l'exorde, la narratio et la confirmatio.

Johann Heinrich Ursin (Ursinus, 1608-1667), Analectorum Rhetoricorum, Sive Sacrae profanaeque Eloquentiae Progymnasmatum libri II, Nuremberg, Michael Endter, 1660, I, chap. 4 à 6, p. 18-29

Suit surtout Théon; mais utilise tous les traités; particulièrement détaillé ; pose comme premier exercice la paraphrase; moyens de développement (ekphrasis, éthopée, etc.), espèces, etc. Intègre à la fabula les emblèmes et les hiéroglyphes. Trois uirtutes seulement. Traite conjointement la chrie et la gnômè ou maxime (chap. 7, p. 30-37). Le livre II fournit des exemples, par exercice, avec notamment d'innombrables versions du Corbeau et du Renard, reprises de divers auteurs, dont Babrius, Phèdre, Camerarius, Apulée...

Surintendant de l'Église de Ratisbonne et pasteur, probablement luthérien.

Pomey, Candidatus rhetoricae, op. cit., p. 257-330, « De narratione et fabula »

Jésuite. Reformule Aphtonius en l'amplifiant à l'aide d'autres traités (non cités), sous forme de dialogue maître-élève.

«De narratione generatim» (« De la narration en général », IV, p. 257). Propose un préambule général commun à la fabula et la narratio, puis les examine successivement. Quatre uirtutes: breuitas, perspicuitas, probabilitas et suauitas («concision, clarté, fiabilité et 'agrément'«), pour lesquelles il reprend les déterminations canoniques (pour la suauitas il suit Part., avec à la fois les uerba et les res, en contaminant Part. 22, 31 et 73) ; consignes, brefs exemples (de narration trop longue, qu'il abrège, notamment); liste de procédés d'expression, ornamenta (proche de Hermogène, mais avec une certaine liberté); liste détaillée avec définition et exemples: incises, présent de narration, infinitifs, etc.; figures: hypotypose, dubitatio, suspensio, communicatio, exclamatio.

Fabula (V, p. 267 sq.) : nombreux détails et exemples; revient sur les quatre uirtutes (p. 276); fournit plusieurs exemples de la même fable (Loup et Agneau, Corbeau et Renard, etc.), traités en styles différents, avec indication des lieux en marge; plusieurs exemples de narrations, $i d$.

À VI, il revient sur la «vraie» narration, "proprement dite (« ueram proprieque dictam» (p. 288) : poetica, historica et ciuilis («poétique, historique et politique»); modes de Diomède pour la narration poétique ou poetica; retour sur les quatre uirtutes: "Suauitatem habeat necesse est, id est, sermonis festiuitatem, colloquia, varios animi motus", "Il faut qu'elle présente de 'l'agrément', de l'enjouement dans l'expression, des dialogues, des attitudes psychologiques variées » (p. 293).

Charles Pajot (1609-1686), Tyrocinium eloquentiae, sive Rhetorica nova, et facilior (1647), Cologne, Johann Wilhelm Friessem II, 1673, III, chap. 5

Jésuite. Paraphrase développée des Progymnasmata d'Aphtonius, avec application à Cicéron. Fabula, narratio, chreia.

Joseph de Jouvancy (1643-1719), Candidatus rhetoricae, Paris, Jean Barbou, 1712, $5^{\mathrm{e}}$ partie "Aphthonii progymnasmata», Progymnasma I «De Fabula», p. 168-194; L'Élève de rhétorique, éd. et trad. D. Denis et F. Goyet, Paris, Classiques Garnier, 2020, p. 258-285

Jésuite. Présentation initiale intéressante; considère la narratio comme un "quasi genus", "quasiment un genre", comprenant la fabula et l'historia comme ses espèces; commence donc par la narratio; à partir de là, il recopie purement et simplement Pomey (voir supra). Passe à la narratio fabulosa ou «narration 
fabuleuse" (voir infra): en réalité, recopie le chapitre Fabula de Pomey, avec quelques exemples différents. Passe directement à la chrie (Progymnasma II, p. 194-253), où il recopie toujours Pomey.

\section{Bibliographie} fiction dans les Progymnasmata d'Aelius Théon ", dans Théorie et pratique de la fiction à l'époque impériale, dir. Christophe Bréchet, Anne Videau, Ruth Webb, Paris, Picard, 2013, p. 37-47.

Aristote, Rhétorique, III, 13 et 16

Énonce la théorie canonique, de façon un peu différente de celle qui deviendra classique chez les Latins, plus proche quant à elle de celle de la Rhétorique à Alexandre. Nuance la notion de brièveté. Distingue les diverses sortes de causes. Insiste sur l'èthos et le pathos, ainsi que sur la proairesis de l'orateur («choix préférentiel »), plutôt que sur des procédés formels. Ne sera vraiment redécouvert qu'assez tardivement, quoique connu au Moyen Âge.

Hermogène, L'invention, II, dans Hermogène, L'art rhétorique, trad. Michel Patillon, Lausanne, L'Âge d'Homme, 1997, p. 223-237 (éd. Rabe, p. 108.20-125.21)

Après le premier livre, sur l'exorde, ce deuxième livre est consacré à «L'exposition (katastasis) ou narration (diègèsis)", selon le titre que lui donne Michel Patillon. Hermogène insiste sur la "prénarration », ou préparation (qui existe aussi chez Quintilien). Conseille de remonter à des états antérieurs, notamment à l'origine. Étudie ensuite longuement la façon de développer la narration. Distingue trois modes. Insiste sur l'acmé de la narration, et son expression stylistique.

Rhétorique à Herennius, I, 12-16 ainsi que Cicéron, De l'invention, I, 27-30, éd. et trad. Guy Achard, Paris, Belles Lettres, 1989 (pour Her.) et 1994 (pour Inv.), respectivement p. $12-15$ et $82-86$

Ces deux textes présentent des développements presque littéralement identiques sur la narratio. Références fondamentales, notamment pour le Moyen Âge. Ils établissent le schéma canonique (nous traduisons). Même définition (Inv., I, 27) : «rerum gestarum aut ut gestarum expositio», "la présentation des événements, 
effectifs ou donnés comme tels "; trois genera ou genres : $1^{\circ}$ portant sur la cause ; $2^{\circ}$ » en dehors de la cause » (" extra causam ») mais en rapport indirect avec elle; $3^{\circ}$ sans rapport avec les débats publics ou « procédures civiles » (" ciuilibus causis »), "delectationis causa non inutili cum exercitatione", "pour le plaisir, non sans profit comme exercice "; deux matières : negotia et personae, " actions" et « personnes". Trois partes pour les negotia : fabula, historia, argumentum, «fable, histoire, 'fiction' ».

Dans une liste importante, sans doute d'origine indirecte aristotélicienne, mêmes procédés principaux pour donner à la narration des personnes la festiuitas, "l'enjouement »: "Hoc in genere narrationis multa debet inesse festiuitas, confecta ex rerum uarietate, animorum dissimilitudine: grauitate, lenitate, spe, metu, suspicione, desiderio, dissimulatione, errore, misericordia, fortunae commutatione, insperato incommodo, subita laetitia, iucundo exitu rerum." "Ce genre de narration doit présenter de l'enjouement ; des retournements de situation, des sentiments divers : sévérité, douceur, espoir, crainte, soupçon, désir, dissimulation, pitié ; changement de fortune, malheur inattendu, joie soudaine, heureux dénouement. » (Inv., I, 27)

Enfin, mêmes uirtutes de la narratio: "ut breuis, ut aperta, ut probabilis ", " concise, claire, fiable » (Inv., I, 28); « ut breuis, ut dilucida, ut ueri similis » (Her., I, 14) ; moyens d'acquérir ces uirtutes, et nuances, surtout pour la breuitas (sur cette notion, voir l'article de Marie Formarier, cité infra). Bornecque, Paris, Belles Lettres, 1960, p. 14-15

Ajoute aux trois uirtutes canoniques la suauitas (Part.32, trad. Bornecque, p. 15): "Suavis autem narratio est quae habet admirationes, exspectationes, exitus inopinatos, interpositos motus animorum, colloquia personarum, dolores, iracundias, metus, laetitias, cupiditates. " «On trouvera de l'agrément aux narrations qui présentent des passages qui étonnent, qui captivent, des dénouements inattendus, par endroit des mouvements pathétiques, des dialogues, de la douleur, de la colère, de la crainte, de l'allégresse, des passions. »

Ce texte des Part. est proche de celui d'Inv. et Her., en fait, où il est question de festiuitas : même source, grecque, probablement, et péripatéticienne. De or., sur la même question, parle de festiuitas (à II, 328), comme Inv, et utilise l'adverbe iucunde (à II, 327, « peruarie iucundeque ») - voir Formarier, art. cit. infra.

On notera que les uirtutes narrationis ont déjà fait leur apparition dans le texte, comme vertus générales du style, en Part. 22, augmentées de l'euidentia-enargeia; les procédés de la suauitas narrative sont en outre repris sous la rubrique ornamenta rerum, à propos de l'éloge, en Part. 73. Voir notre autre article dans cette même livraison.

Pour les commentaires humanistes de ce texte, voir infra.

Cicéron, Topiques, 97, éd. et trad. Henri Bornecque, Paris, Belles Lettres, 1960, p. 100

Liste de six uirtutes : « ut planae, ut breves, ut euidentes, ut credibiles, ut moratae, ut cum dignitate », " claires, concises, imagées, fiables, naturelles, pleines de bienséance et de noblesse » (trad. Bornecque modifiée); soit, dans la trad. Jean Cousin de ce passage cité par Quintilien, narration « toute unie ", "brève ", " plausible », qu'elle ait « l'évidence », enfin « la convenance avec la dignité » (ou dans la trad. de l'abbé Gedoyn : « convenable aux mœurs \& au caractére des personnes, \& soustenuë d'une certaine dignité », De l'institution de l'orateur, Paris, Grégoire Dupuis, 1718, p. 245). Ce passage de Top. 97 est rarement repris, mais quelquefois quand même, par exemple chez Érasme. Quand Quintilien (IV, 2, 64) cite cette liste de six uirtutes, en écrivant lui aussi « morata», c'est pour la récuser, comme on va le voir à l'instant.

Quintilien, Institution oratoire, IV, 2 ; éd. et trad. J. Cousin, op. cit., t. III, 1976, p. 39-74

Reprend le schéma canonique, mais de façon très détaillée et nuancée, avec beaucoup de compléments, notamment sur l'enargeia ou euidentia (non nommés, 
mais bien présents aux $\$ 123-124$ : il faut « ajouter à la vérité un tableau plausible des faits, qui donne aux auditeurs l'impression qu'ils assistent, pour ainsi dire, à la scène »), ainsi que sur la persona de l'orateur (\$ 125-126, « tout le crédit qu'apporte à un exposé l'autorité du narrateur »), etc.

Précise que, selon certains, il faut ajouter aux trois uirtutes canoniques la

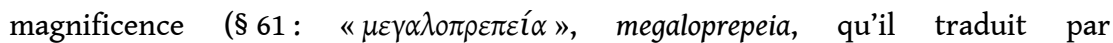
«magnificentia»), et qu'il convient aussi, selon Théodecte, que la narratio soit iucunda ( $\$ 63$ : « iucundam expositionem », " non seulement de la magnificence, mais aussi de l'agrément », trad. Cousin). Il ajoute alors (\$ 64) une critique détaillée de Top. 97 : «Cicéron demande encore un plus grand nombre de qualités. Il veut que la narration soit toute unie, brève et plausible, mais aussi qu'elle ait l'évidence, la convenance avec la dignité. Mais dans le discours, tout doit avoir, autant que possible, ce ton de convenance avec la dignité ; quant à l'évidence dans la narration, pour autant que je comprenne le terme, c'est, à vrai dire, une grande qualité, quand il faut non pas tant dire que montrer du doigt [ostendendum], en quelque sorte, ce qui est vrai ; toutefois, on peut la faire entrer dans la transparence [perspicuitatis] [...]. » latini minores, éd. Karl Halm, Leipzig, Teubner, 1863, p. 155-304

Nous renvoyons ici à nos remarques sur l'article (en ligne) de Paula Olmos Gómez, cité infra dans la bibliographie.

Fortunatien (Fortunatianus), Ars rhetorica, II, § 16-20, dans Rhetores latini minores, op. cit., p. 110-113 ; Consulti Fortunatiani Ars Rhetorica, éd. et commentaire par Lucia Calboli Montefusco, Bologne, Pàtron, 1979

Développement extrêmement détaillé et d'une extrême importance. Sera souvent repris au Moyen Âge, et largement exploité encore par la suite, pendant toute la Renaissance. Multiplie les subdivisions, avec lexique technique grec et latin : cinq genera ou genres, huit species ou espèces (reprises par Caussin, voir S. Duval et L. Charles dans le présent numéro). Insiste sur la praeparatio ou "exposé préalable ». Donne des consignes d'usage de la narration concisa (p. 111, 1.16-21 et p. 112, 1. 30), non pas « concise» mais « fragmentée, interrompue» (littéralement " coupée, disloquée »), pour répondre d'emblée aux points les plus scandaleux de celle de l'adversaire (la concisa correspond à l'intercisa du $\$ 10$ de Vossius, voir infra). Virtutes: breuis, manifesta, uerisimilis ("brève, claire, vraisemblable »). Figures :

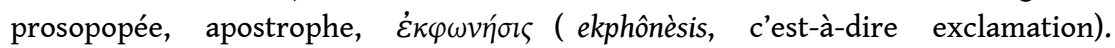
Digression (excessus), etc.

Le succès de cette Ars rhetorica est attesté par l'existence de deux incunables, «le Braidense, sans doute édité à Milan aux environs de 1484 et considéré comme l'editio princeps et l'Ambrosiano, copie légèrement postérieure. Puis vient l'Aldine de 1523, réimprimée à Bâle en 1526 et à Louvain en 1550, suivie de celle de Pierre Pithou en 1599 qui deviendra la vulgate » (résumé des pages 39-46 de l'introduction de L. Calboli Montefusco, par Edmond Liénard dans son compte rendu, L'Antiquité classique, 1980, $\mathrm{n}^{\circ} 49$, p. 424).

p. $322-323$

Assez rapide, mais des éléments de détail qui sont parfois repris par les humanistes. Trois uirtutes usuelles.

Martianus Capella, De nuptiis philologiae, \& septem artibus liberalibus libri nouem, Lyon, héritiers Simon Vincent, 1539, V «De rhetorica», p. 208-209 (pages intitulées «De narratione ») ; titre et références actuels, De nuptiis Mercurii et Philologiae, V, § 550-552

Bref, mais intéressant. Reprend la doctrine usuelle des trois uirtutes, mais introduit des précisions et des distinctions inhabituelles, notamment sur le caractère continu 
ou non de la narration, et sa possible apparition dans le corps de la harangue. Reprend les distinctions grecques de Théodore de Byzance, celles mêmes qu'Aristote conteste en Rhétorique, III, 13. Texte bien connu des médiévaux, de surcroît. Indispensable, donc.

La dernière édition du texte latin est celle de James Willis (Stuttgart, Teubner, 1983). Pas encore de traduction française du livre V, apparemment: les Belles Lettres (C. U.F.) ont publié les livres I, IV, VI, VII et IX. Sont disponibles une traduction en anglais, par William Harris Stahl et al., Martianus Capella and the Seven Liberal Arts, vol. II, The Marriage of Philology and Mercury, New York, Columbia University Press, 1971, et une autre en allemand, par Hans G. Zekl : Martianus Capella. Die Hochzeit der Philologia mit Merkur, Würzburg, Königshausen/ Neumann, 2005.

\subsection{Moyen Âge}

Brunetto Latini (1220-1295), Li livres dou Tresor, III, I, chap. 39-42, Paris, Imprimerie Impériale, 1863 , p. 517-523

Reprend en les détaillant Inv. et Her. ; est une des principales sources de Fabri. Rend narratio par « Fait ». Est sans doute la première rhétorique en langue française, très complète. Trois «choses: que ele soit brieve, que ele soit clere, que ele soit voirsemblable » (p. 519). Par ailleurs, traite du « conte », de façon plus générale, en entendant par là l'oratio tout entière, mais avec des applications au récit, en fait (p. 482 sq., chap. 11, « de l'ordre »). Mentionne en particulier la liste canonique des huit procédés d'amplification, systématique dans toutes les poétiques et rhétoriques médiévales, jusqu'à Fabri compris. Édition critique disponible, par Francis J. Carmody, Berkeley/Los Angeles, University of California Press, 1948 (Genève, Slatkine repr., 1998). À voir pour sources.

Jacques Legrand (Jacobus Magnus, 1360 ?-1425 ?), Archiloge Sophie, Livre de Bonnes Meurs, 19, éd. Evencio Beltran, Paris, Champion, 1986, p. 122

Très succinct.

Georges de Trébizonde (Georgius Trapezuntius, 1396-1486), Rhetoricorum libri V (ca. 1472), Bâle, Valentin Curio, 1522, I « De narratione », fo $10 \mathrm{r}^{\circ}-15 \mathrm{r}^{\circ}$

Développement très détaillé, avec de nombreuses analyses, surtout de Cicéron, mais aussi de Virgile. Le schéma général est celui de Inv. et Her.; mais introduit de nombreuses distinctions secondaires, quant au type de cause, selon l'accusation ou la défense, etc. Virtutes usuelles. S'attache particulièrement au dosage des affects, qui doivent être en principe modérés, à la différence de la péroraison, mais avec des exceptions, en particulier dans le cas de mouvements véhéments occasionnels dits procursus. Développement de première importance. Autres développements: au livre IV, rapide, sur la narratio dans le genre démonstratif ( $\left.f^{\circ} 101 \mathrm{r}^{\circ}\right)$ et ( $\left(^{\circ} 110 \mathrm{r}^{\circ}\right)$ dans le cadre de la dispositio, avec exemple de Démosthène. Et, au livre $\mathrm{V}\left(\mathrm{f}^{\circ} 173 \mathrm{r}^{\circ}\right.$ $\mathrm{v}^{\circ}$ ) : sur la forma adaptée à la narratio ; plutôt style moyen, pas trop orné, avec une certaine force affective, cependant, modérée, sauf dans les procursus (les mouvements véhéments, comme défini plus haut).

Guillaume Fichet (Fichetus, 1433-ca.1480), Rhetorica, s. 1.n.n. [Paris, Ulrich Gering, Martin Crantz et Michael Friburger], 1471, n. p. (vues 157-169 de l'exemplaire numérisé par la BnF, NUMM-8713311, sur Gallica)

Développement très important ; reprend Inv. et Her., mais avec des précisions non négligeables, notamment sur la façon d'adapter la narratio au type de cause. Doit dépendre en partie de Trébizonde, qu'il connaissait.

Guillaume Tardif (1440-1492), Rhetorica, Paris, s. n. [Au soufflet vert (Louis Symonel)], $1475, f^{\circ} 4 v^{\circ}-6 r^{\circ}$ 
Développement dans le même esprit que Fichet, mais différent dans le détail. Johann Gymnich (Gymnicus), 1539 ; éd. et trad. allemande de Lothar Mundt, Tübingen, Max Niemeyer, 1992 ; éd. et trad. française (partielle) de Marc van der Poel dans Écrits sur la dialectique et l'humanisme, Paris, Champion, 1997

Les deux éditions modernes renvoient aux pages de l'édition 1539 (qui a les copieux commentaires d'Alard d'Amsterdam). Au chapitre 16 du livre II (p. 258-260, Mundt p. 302-306, Poel p. 136-145), Agricola envisage la narratio, simple partie du discours, dans le cadre général de ce qu'il appelle l'expositio (présentation brute des idées, sans volonté de persuader) et l'argumentatio, indissociables. Analyse de l'Énéide, dialectique (p. 262, Mundt 1. 26-38). Au chapitre 22 du livre II (p. 296-301, Mundt p. 344-352, Poel p. 162-171), développe fortement l'expositio; vraie ou fausse; exemples; deux qualités (n'utilise pas la terminologie des uirtutes) : probabilis et accommodata, «fiable » et "adaptée » c'est-à-dire adaptée à l'auditoire (p. 298, Mundt, 1.60-64 et 70-76); déterminations du probabilis: 1/argumentosa ou «argumentée », 2/ consentanea rebus ou "cohérente avec les faits» 3/per se consequens ou «enchaînée logiquement », soit 1/ présenter les causae (« origines de la situation »), 2/ personnes, choses, lieux, temps, 3/ cohérence rigoureuse (avec exemples du Pour Milon et de l'Énéide); Cicéron, Térence; allers et retours sur les événements, créer l'attente (p. 298-301, Mundt, p. 350-352). Au chapitre 4 du livre III (p. 394-399, Mundt p. 454-464), revient sur la delectatio, dont il fournit des déterminations proches de celles de la suauitas dans Part.: «Mentem [...] delectant omnia quae magna, admiranda, inopinata, inexpectata, inaudita sunt [...]», "Ce qui procure du plaisir à l'esprit [delectant], c'est tout ce qui est important, qui étonne, qui est inattendu, qui captive, qui est inouï » (p. 395, Mundt, 1. 41); « habeat oratio motus animorum, colloquia personarum, consilia, euentusque rerum inopinatos », « il faut que le discours ait des passages pathétiques, des dialogues, des desseins inattendus, tout comme les événements qui en résultent » (p. 396, Mundt, 1. 106-107), tout cela étant à mettre en scène avec art. Ensemble atypique, même s'il y a reprise subreptice des données canoniques. Influence capitale pour la tradition mélanchthonienne et ramiste. Probablement aussi ailleurs, de façon diffuse (en particulier pour la suauitas).

\section{3. $\mathrm{XVI} \mathrm{e}^{\mathrm{e}}$ siècle}

Érasme (1466/69-1536), De duplici copia (1512), II, "De fictis narrationibus », éd. Betty I. Knott, dans Érasme, Opera omnia, Amsterdam, North-Holland, t. I-6, 1988, p. 256-269, LB 99E-105E (LB : pages des Opera omnia, Leyde, Van der Aa, 1703-1706, t. 1)

Développement très détaillé sur les "narrations fictives» (incipit: «Iam fictae narrationes rerum gestarum... »). Propose surtout une taxinomie des sujets ou des genres (apologue, parabole, etc.), et une liste de sources possibles de l'invention (tragédies, textes d'histoire naturelle, etc.). - Traduction française partielle du De copia (La double abondance des mots et des idées) dans Érasme, Euvres choisies, trad. Jacques Chomarat, Paris, Librairie Générale Française («Le Livre de poche classique »), 1991, p. 233-258, dont la fin de ce passage (103E-105E), p. 250-256. Voir aussi, ci-dessous, la rubrique Narratio fabulosa.

Id., Ecclesiastes sive de Ratione concionandi (1535), II, dans Érasme, Opera omnia, Bâle, Hieronymus Froben, 1540, t. V, p. 721-723 ; éd. Jacques Chomarat, dans Érasme, Opera omnia, Amsterdam, North-Holland, t. V-4, 1991, p. 286-290, LB 865C-867A (LB : pages des Opera omnia, Leyde, Van der Aa, 1703-1706, t. 5)

Développement plutôt original (à partir de "Vehementius autem afficit narratio" jusqu'à «qui ficta pro veris narrant»). Virtutes: la narratio "produira un effet de véhémence » (p. 286, 1. 891) «si sit probabilis », «si ordine texatur », «si sit euidens », 
"si decorum seruet ", soit « si fiable, si disposée en ordre, si imagée, si conforme à la bienséance ». Ne correspond pas exactement au sens cicéronien des termes, du moins dans Inv. et Part., mais évoque les six uirtutes de Cicéron, Topiques 97 (voir cidessus). Érasme explicite probabilis par "si naturae ac mori respondeat », " rendant compte de la nature et des mœurs »; euidens par « rem ueluti depictam », « mettant la chose sous les yeux", c'est-à-dire l'enargeia; decorum par "si decorum seruet in personis, \& in sermocinationibus ac dialogismis ", "si on respecte le caractère dans les personnes et leurs propos ». Insiste sur les èthè (éd. 1991, p. 286, 1. 907 sq.), et les différences entre les personnages, et même les animaux. Est un véritable exposé synthétique de l'esthétique spécifiquement érasmienne de la narration. Traduction française partielle de l'Ecclesiastes (Le Prédicateur), mais sans ce passage, dans Érasme, Euvres choisies, op. cit., p. 974-1021.

Pierre Fabri (Pierre Le Fèvre, 1450 ?-1535 ?), Le grant et vray art de plaine rhetorique éd.

Alexandre Héron, Rouen, Cagniard, 1889-1890, t. 1, p. 64

Développement détaillé, qui reprend Inv. et Her., mais en détaillant dans un esprit proche de Fichet et Tardif; Héron identifie comme sources fréquentes Brunetto Latini, surtout, Fichet et Tardif, sans parler de Inv. et Her. Sur la narration, Fabri suit en réalité de très près Latini, dont il modernise la langue, mais avec certaines variantes, et une tendance générale à abréger. Parle de «narration » (alors que Latini emploie le mot « fait »).

Dubois, F. Sylvii Ambiani in orationem M. T. Ciceronis pro Milone commentarii, recogniti \& aucti : preposito de arte dicendi in genere judiciali compendio (1526), Paris, Josse Bade, 1531, $\mathrm{f}^{0}$ alII vo-aiiii

Applique les consignes rhétoriques de la narratio à celle du Pour Milon. Insiste sur les status ou «états de cause ", l'amplification et les trois uirtutes (d'après Quintilien). Des éléments intéressants aussi dans le corps du commentaire proprement dit, sur la narratio, mais l'essentiel est résumé dans le compendium liminaire.

Ringelberg (Joachim Sterck van-, 1499-1531 ?), Joachimi Fortii Ringelbergii Antverpiani rhetorica, nunc demum tersißimè emendata, Troyes, Nicole, 1542, p. 16-17

Assez détaillé sur la narratio et la digressio, et original dans ses divisions, avec un plan proposé, et des analyses d'exemples. - Sur cet auteur, voir la notice de Peter Mack, A history..., op. cit., p. 124-125.

41 Melanchthon (1497-1560), De Rhetorica libri tres (Wittenberg, 1519), Bâle, Johann Froben, 1519

Livre I : lieux de la narratio dans l'éloge, p. 50 ; plan type; exemple : dans le genre délibératif, p. 79 (sommaire); dans le genre judiciaire, p. 97 (sommaire, insiste sur l'argumentation); analyse du Pour Milon (p. 99), mais du point de vue du status conjecturalis (" état de cause conjectural ») ; liste de lieux. Livre II «De dispositione », p. 108 : suivre l'ordre des faits, et l'ordre logique; insistance sur le syllogisme; analyse du Pour Milon en termes dialectiques (p.112-113), réduction à l'ordre syllogistique. Volkhard Wels, Berlin, Weidler, 2001

Succinct, insiste surtout sur son absence dans les genres délibératif (I, p. 56, Wels p. 118) et même souvent judiciaire (p. 34, Wels p. 70-72); un peu plus long à propos de la narratio dans le genre démonstratif (p.62-63, Wels p.130-132). Les considérations sur la dispositio de la narratio sont très brèves et sommaires (p. 74, Wels p. 156). Au livre II, un paragraphe rapproche narratio et color: le color « n'est pas une figure », mais « une sorte de figure, le mot désigne en effet une narratio inventée de façon vraisemblable, quand par exemple nous imaginons ce que quelqu'un a pu penser, dire ou faire", et "les poètes sont pleins de ce genre de narrations, parce qu'ils décrivent des fictions » («color est species figurae, significat 
enim narrationem uerisimiliter fictam, ut cum fingimus, quid aliquis opinetur, quid dixerit, quid fecerit ", "Poëtae pleni sunt huiusmodi narrationibus, quia res fictas describunt", p. 126-127, Wels p. 262). - Pour la date de la première édition, 1531, nous reprenons Wels, p. 465.

Id., Institutiones Rhetoricae (1521), Strasbourg, s. n. [Christian Egenolff], 1523

$\mathrm{F}^{\mathrm{o}} 6 \mathrm{r}^{\mathrm{o}}$ (utilisation rare dans l'éloge des personnes) ; $6 \mathrm{v}^{\mathrm{o}}:$ id., éloge des choses $; 7 \mathrm{v}^{\mathrm{o}}$ : plus détaillé, genre délibératif ; $9 \mathrm{r}^{\circ}$ : judiciaire, un peu plus détaillé. Très succinct dans l'ensemble. Simple compendium. ratio ad usum forensem... (1536), Cologne, Eucharius Cervicornus, 1538

Appliqué au droit. Doctrine canonique, d'après Quintilien, avec peu de variantes.

Johannes Sturm (1507-1589), De amissa ratione dicendi (1538), Lyon, Gryphe, 1542

Commentaire du Pour Quinctius de Cicéron, en particulier de sa narratio, conduit dans l'esprit de la dialectique d'Agricola, notamment. D'un très grand intérêt.

Johannes Caesarius (Giovanni Cosenza, 1468?-1550?), Rhetorica in septem libros... (1534), Lyon, Thibaud Payen (Theobaldus Paganus), 1542, V, chap. 6 «De narratione» et 7 «De tribus narrationis uirtutibus siue laudibus », p. 77-80

Cite Cicéron, Martianus Capella, Trébizonde. Reprend globalement Inv. et Her., mais avec analyses d'exemples, en particulier poétiques (Ovide), et des distinctions de détail. - Sur cet auteur, voir la notice de Peter Mack, A history..., op. cit., p. 104-106.

Pierre de Courcelles (dates inconnues), La Rhetorique, Paris, Guillaume Le Noir I, 1557

En fait adaptation française de celle de Caesarius, mais avec exemples tirés de poètes français, dont la Pléiade. $\mathrm{F}^{\mathrm{o}} 3 \mathrm{v}^{\mathrm{o}}$ : succinct; $20 \mathrm{v}^{\mathrm{o}}$, chapitre 6 : «De la narration, recit ou conte "; long développement pour l'essentiel traduit d'Inv. et Her., mais avec des exemples en vers français très nombreux et riches.

Jacques Amyot (1519-1593), Projet de l'éloquence royale, composé pour Henry III, roi de France, Versailles, Pierres, 1807, chap. 11, p. 37 ; éd. P.-J. Salazar, Paris, Les Belles Lettres, 1992

Sommaire ; «vraisemblable, éviter prolixité, fuir l'obscurité [...] en gardant l'ordre des choses telles qu'elles sont advenües » (p. 38); peut être parfois omise.

Giason Denores (Giasone de Nores ou Jiasone Nores de Cipro, ca. 1530-1590), Della rhetorica libri III, Venise, Paolo Meietti, 1584, II «Dell'ordine delle questioni, et della narratione », p. 80

Succinct; trois uirtutes; quelques exemples. N'envisage la narration que dans le cadre de l'argumentation.

Bartolomeo Cavalcanti (1503-1562), Della retorica (1558), Venise, Giovanni Giolito de Ferrari, 1559, VI, p. 431-452

Développement très important consacré à la narration (traite la proposizione auparavant). A partir des trois uirtutes, mais très détaillé, avec exemples des auteurs, ou illustrations personnelles. Nuance la brevità. Perspective largement dialectique, avec lieux de l'argumentation. Traite de près les costumi (c'est-à-dire les caractères ou èthè) et les affetti (les pathè). Types de narration selon les genres rhétoriques, et les constitutions de causes. Ajoute « una certa ampiezza, vivacità \& leggiadria ", " une certaine ampleur, vivacité et élégance ", où les déterminations de la suauitas de Part. sont contaminées avec celles de la festiuitas d'Inv. et Her. (p. 436). Influence probable d'Agricola. - Sur cet auteur, voir la notice de Peter Mack, A history..., op. cit., p. 172-176. 
51 Francesco Robortello (1516-1567), De artificio dicendi, Bologne, Alessandro Benacci (Benatius), 1567, fo $35 \mathrm{r}^{\circ}$

Développement d'esprit aristotélicien, original pour l'époque; èthikè rendant le caractère; figures; rôle central de la proairesis aristotélicienne («choix préférentiel »).

Francesco Sansovino (1521-1586), L'arte oratoria secondo i modi della lingua volgare, Venise, Giovanni Griffio I (Gryphius), 1546, fo $12 \mathrm{r}^{\circ}$

Triple division d'Inv. et Her. ; ordre naturel / artificiel ; reprend les divisions d'Inv. et Her., mais en subdivisant, et en appliquant à la littérature italienne ; développe sur l'histoire, mentionnant les annales et les chroniques, latines et italiennes. Trois uirtutes. Proche d'Inv. et Her., avec peut-être un peu de Quintilien, mais exemples italiens, surtout de Pétrarque. - Sur cet ouvrage, voir le paragraphe de Peter Mack, A history..., op. cit., p. 301-302.

Germain Forget (mort en 1610 ?), Rhetorique françoise faicte particulierement pour le Roy Henry III, Precetti di rettorica scritti per Enrico III re di Francia, éd. Giulio Camus, Modène, Antica Tipografia Soliani, 1887

S'interrompt après avoir traité l'exorde... Peut-être optique ramiste, qui privilégie l'elocutio (le style), mais cela se discute, puisque la dispositio est traitée - en second, il est vrai, après les figures - mais de façon brusquement interrompue. Alex Gordon date de 1583 (environ) l'un des deux manuscrits connus, celui de Carpentras (« Les figures de rhétorique au $\mathrm{XVI}^{\mathrm{e}}$ siècle ", L'Information grammaticale, $\mathrm{n}^{\circ} 75$, oct. 1997, p. 15, n. 4). Giulio Camus a édité l'autre manuscrit connu, celui de la Biblioteca Estense de Modène.

Francesco Patrizi (1529-1597), Della retorica... (1552), Venise, Francesco Senese, 1562

Très général. - Sur cet ouvrage, voir le paragraphe de Peter Mack, A history..., op. cit., p. 304.

Pierre Saint-Fleur (ou Sainct-Fleur, dates inconnues), Institutionum rhetoricarum libellus, Paris, Thomas Brumen (Brummenius), 1561, chap. 21 « Narratio », fo $29 \mathrm{~V}^{\circ}$

Série de citations des auteurs (Cicéron, Hermogène, Quintilien), avec commentaire souvent détaillé, et exemples, en particulier de textes de fiction (Ovide) ; intéressant par le choix des références. Ouvrage dédicacé à Amyot. Reprend la suauitas, d'après Cicéron, Part. (premier exemple par nous repéré au $\mathrm{XVI}^{\mathrm{e}}$ siècle); mais l'interprète seulement en termes d'élocution (éviter les rencontres fâcheuses de sons, bien organiser la structure des phrases), en reprenant pour l'essentiel Part. 22; taxinomie des types de narration, selon Quintilien et Hermogène, détaille (éloges, descriptions, etc.).

Nicolaus Biesius (Nicolas Biese, 1516-1572), De arte dicendi libri II, Anvers, Philippe Nuyts (Nutius), 1573, II, fo $57-58$

Reprend Inv. et Her., mais avec exemples de Cicéron analysés.

57 Cipriano Soarez (Súarez ou Soarius, 1524-1593), De arte rhetorica libri tres. Ex Aristotele, Cicerone et Quinctiliano praecipue deprompti (1557), Brescia, Tommaso Bozzola, 1581, II, chap. 8 « De narratione » et 9 "Quando narratione utendum sit », fo $31 \mathrm{v}^{\circ}-\mathrm{f}^{\circ} 32 \mathrm{r}^{\circ}$

Jésuite. Exposé condensé, solidement scolaire ; trois uirtutes ; abrège plus ou moins Quintilien, mais avec des emprunts à Cicéron et Aristote. L'ouvrage est un classique de l'enseignement jésuite, il est préconisé par le Ratio studiorum. - Sur cet ouvrage, voir la notice (et le plan) de Peter Mack, A history..., op. cit., p. 177-182.

Agostino Valiero (Valerius, 1530-1606), De rhetorica ecclesiastica (1574), Paris, Thomas Brumen (Brummenius), 1575 (l'édition de Louis de Grenade que nous citons juste après contient aussi cette œuvre) 
Rien, ou presque. Aucune place pour la narratio dans le sermon tel que l'entend cet évêque de Vérone. - Sur cet ouvrage, voir les deux paragraphes de Peter Mack, A history..., op. cit., p. 268-269. section II « De Narratione », Venise, Francesco Ziletti, 1578, p. 141-147; La Rhétorique de l'Eglise, ou l'Eloquence des Prédicateurs, trad. Nicolas-Joseph Binet, Paris, Jean Villette, 1698 , IV, ch. 1, section II « De la Narration, \& sur tout des quatre sortes qui servent à la Prédication », p. 6-10

Adapte la narratio à l'éloquence sacrée; distingue quatre espèces propres à ce genre ; quatre uirtutes, dont iucunda (p.141C; «les Rhéteurs [...] veulent que toute Narration soit courte \& claire \& qu'il y ait de la vray-semblance \& de l'agrément ", p. 7), avec spécifications canoniques de la suauitas («Enfin la Narration a de la douceur \& donne du plaisir, quand elle contient des choses grandes \& importantes, des choses curieuses, extraordinaires \& toutes nouvelles, ausquelles on ne s'attend point ", p. 9). - Sur cet ouvrage, voir la notice (et le plan) de Peter Mack, A history..., op. cit., p. 269-272.

Alonso Garcia Matamoros (Alphonsus Garsias y Matamoros, 1490-1550), De ratione dicendi libri duo (1548), Alcalá de Henares, Andrés Angulo, 1561, I, chap. 11 et 12, fo $31 \mathrm{v}^{\mathrm{o}}-38 \mathrm{r}^{\mathrm{o}}$

Développement très ample, et relativement original, notamment dans l'énoncé des uirtutes, avec de nombreux exemples analysés, et une attention particulière pour les lieux, dans une perspective qui est surtout celle de l'éloge.

61 Lodovico Carbone (Ludouicus Carbonus, mort en 1597), De causis eloquentiae libri IIII (1590), Venise, Damiano Zenario, 1593, III, p. 357

Jésuite. Rapide, sur la dispositio. Introduit l'ordo artificialis / naturalis, l'« ordre artificiel / naturel ». Aborde plus loin le début in medias res (voir infra). Son De arte dicendi de 1589 (chez le même éditeur) est encore plus succinct: Carbone rappelle surtout la position aristotélicienne (Rhétorique, III, 13) selon laquelle la narratio tout comme l'exorde n'est pas de l'essence du discours (Tabulae rhetoricae Cypriani Soarii, sacerdotis e Societate Iesu : qvibvs accesservnt dvo libri De Arte dicendi, II, p. 102, 112 et 118).

Id., Diuinus orator vel de rhetorica diuina libri VII, Venise, Societas minima, 1595, III, 2, p. $118-120$

Assez long développement. Reprend le schéma canonique, mais en l'enrichissant de la quatrième vertu des Part., la suauitas, et en l'appliquant aux sujets chrétiens et à l'Écriture. Signale au passage la qualité morata, c'est-à-dire le fait de «bien peindre les caractères ». Très sensible à la valeur argumentative en vue de l'éloge.

Melchior Junius (Johann Sommer, 1545-1604), Methodus eloquentiae comparandae (1585), Bâle, Konrad von Waldkirch, 1589, p. 155

Professeur à Strasbourg. Se contente de conseiller à l'apprenti orateur de se constituer une collection d'exemples de narrations tirées de Cicéron. Ne donne aucune consigne. - Sur cet auteur (et cet ouvrage), Peter Mack a quelques lignes assez sommaires (A history..., op. cit., p. 162).

Id., Artis dicendi praecepta secundum oratorii officii partes breuiter ex Platone, Aristotele, Hermogene, Cicerone, Herenniano magistro, Quintiliano congesta ac digesta (1589), Strasbourg, Anton Bertram (Bertramus), 1590, p. 295-303

Exposé détaillé, assez classique, mais avec des sources assez riches, notamment grecques. Utilité ou pas de la narratio. Virtutes : breues, probabiles, apertas, suaues, moratas ("concises, fiables, claires, 'agréables', peignant bien les caractères »). Chacun des termes sert de manchette à un paragraphe qui donne détails et 
références. Ceux sur la breuitas sont liés au point à choisir pour commencer. Sur la probabilitas: dialogues, description des gestes, etc. Sur la suauitas: suspens (exspectationes), affects, etc., le tout provenant de Part. Sur morata (retraduit en grec par « $\left.\dot{\eta} \theta_{\imath} \kappa \dot{\eta} », \grave{t} t h i k \grave{e}\right)$, cinq procédés, dont l'indication du dessein, de la uoluntas etc. c'est-à-dire de la proairesis («choix préférentiel») d'Aristote, qu'il reprend; ou encore "par le fait de rendre acceptables des choses incroyables ou absurdes" (" probatione [...] incredibilium vel absurdorum », p. 300). Enfin, moyens de dilater la narration (tiré d'Hermogène) et vices de la narration. conscribendis orationibus, e veterum ac recentiorum oratorum praeceptis methodica introductio (1613), Amsterdam, Johannes van Ravesteyn, 1654, chap. 4, p. 25-30, « Narratio »

Ramisme modéré. Assez riche; cinq uirtutes: breuis, perspicua, probabilis, suauis, methodica; assez classique sur les uirtutes usuelles (Part., etc.) ; «methodica» veut dire surtout «suivre l'ordre des faits »; listes de lieux; exemples, en particulier de narratio methodica. - Sur cet auteur (et cet ouvrage), voir la notice de Peter Mack, A history..., op. cit., p. 196-198.

Nicolas Caussin (1583-1631), De eloquentia sacra et humana libri XVI (1619), Paris, Guillaume Pelé, 1637, VI, p. 324

Jésuite. Voir S. Duval et L. Charles dans le présent numéro. Développement extrêmement détaillé - en réalité recopié en grande partie du commentaire de Part. par Majoragius -, qui envisage toutes les sortes possibles de narrations, fournit des espèces à l'infini, en s'appuyant sur la rhétorique grecque, notamment. Propose une liste de huit uitia (vices de style) et les trois uirtutes usuelles. Pour la probabilitas, ses 15 divisions sont autant de lieux. Ajoute la suauitas, avec 10 divisions, reprises de Part., et exemples. Exemples: de narrationes poeticae (Libanios, etc.; emprunt à Lorichius, il me semble); de narratio historica (Élien); politica (Cicéron); pulchra ("Narratio et hypotyposis pulchra», exemple de "narration et hypotypose remarquable» chez Quintilien, Controuersiae). Autres exemples (Platon, Cicéron, etc.). Est donc une petite somme sur la narration. Privilégie des exemples de style 
fleuri : on croirait lire du Fénelon à l'avance... - Sur cet ouvrage, voir la notice (et le plan très détaillé) de Peter Mack, A history..., op. cit., p. 198-206s.

\section{partitionum oratoriarum libri quinque (1621), Leyde, Joannes Maire, 1640, III «De} dispositione », chap. 5 « De narratione », p. 268-276

Évoque la narration résumée ou "exposé préparatoire» («praeludium»,

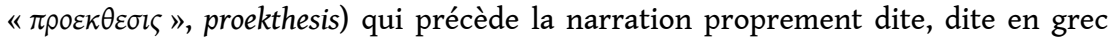
ekthesis $(\S 2)$; deux premières espèces seulement ; subdivision : continua / intercisa, « continuée » / « fragmentée » (exemples de Cicéron, § 7-11; l'intercisa correspond à la concisa de Fortunatien, voir supra et ici le $\S 10$ ). Virtutes : perspicua, verisimilis, suauis, «claire, vraisemblable, 'agréable'«. Sur suauitas reprend Part. Renvoie aux Éthiopiques, néanmoins un peu affectées, selon lui. Rejette la breuitas. Digressio (renvoie au chapitre qu'il consacre à la digression : IV, chap. 19, p. 405).

Professeur à Leyde. Calviniste libéral. Antiramiste : attaque les antiperipatetici dans son De rhetorices natura ac constitutione, et antiquis rhetoribus, sophistis ac oratoribus liber, Leyde, J. Maire, 1621. Peter Mack (A history..., op. cit., p. 192-196) a une notice très développée, avec plan, sur la « grande » rhétorique de Vossius, les Institutiones oratoriae de 1606, dont la Rhetorices contractae... est une sorte de version abrégée.

Pajot, Tyrocinium eloquentiae, op. cit.

À la page 152, associe à la narratio les figures du pathos, c'est-à-dire " plus aptes à susciter les réactions affectives» (" aptiores ad concitandos affectus») : cela vient de Macrobe, en plus réduit, mais mérite d'être signalé, car rare, et avec exemples. Le chapitre «De narratione» est le deuxième du livre III, p. 186: deux espèces seulement (oratoires); praeparatio, «exposé préalable»; liste de procédés syntaxiques et de figures (hypotypose, suspensio ou "suspension », etc.). Exemples de Cicéron. Quatre uirtutes: probabilitas, breuitas, suauitas, perspicuitas, "fiabilité, concision, 'agrément', clarté ». Détaille, avec exemples de Cicéron. Sur la suauitas reprend Part. 21-22 et 31, en 8 rubriques (expression et contenu) ; utilise Majoragius ou Caussin, mais ne les suit pas; insiste sur les épilogues; la narratio doit être festiua. Digressio. Consignes de composition (assez générales : s'en tenir au fait).

Gérard Pelletier (1587-1648), Reginae Palatium Eloquentiae (1641), Lyon, Jean-Aimé Candy, 1653

Jésuite. Le livre V débute (chapitre ou «lectio » 1, p. 251) par un exposé général sur la dispositio ; signalons (p. 265, paragraphe ou «punctum » 24) un exorde tiré d'une fabula (exemple poétique). Les pages sur la narratio sont V, chap.10, p. 288-300. Deux espèces, reprises de Quintilien ; praeparatio (Hermogène), sinon description (exemple d'Ovide) ; ornements ( Hermogène, etc.) ; figures : hypotypose, suspensio, etc. (exemples); motus; quatre uirtutes (dont la suauitas) ; conditions des uirtutes, très développé. Pour la suauitas, repris de Part., avec additions, soit au total 10 rubriques, différentes de celles de Caussin/Majoragius: exclamations, infinitifs, apologues, histoire... ; festiuitas (rubrique 8, contamination d'Inv., Her. et Part.), avec développements et exemples. Pour la narratio personarum, intègre la festiuitas et ses moyens (repris d'Inv. et Her.) ; considère l'egressio ou digression comme une uirtus plutôt que comme une pars, une partie et non une qualité (exemple, bouclier d'Énée, etc). Dans le livre X, p. 857, examine à nouveau la conduite de la narratio, à propos du genre judiciaire, situation par situation et sur des exemples (mais assez sommaire); là où cet exposé est le plus développé, c'est à propos de l'apologia ou " apologie ", avec exemples divers dont la narration du Pour Milon.

René Bary (mort en 1680), La Rhétorique françoise où pour principale augmentation l'on trouve les secrets de nostre langue (1653), Paris, Le Petit, 1660, p. 266

Cinq sortes: "judiciaire», "digressive», "fabuleuse », " romanesque», "prophétique»; division étrange, mais qui correspond probablement aux trois espèces d'Inv. et Her., où la narration delectationis causa (« pour le plaisir ») est 
subdivisée en fabula, la «fable » (au sens large, soit « fabuleuse » chez Bary), historia (l'histoire véritable, ici sans doute le "judiciaire ») et argumentum, la "fiction » (correspondant ici au « romanesque »). La catégorie du " prophétique » semble être $\mathrm{du}$ cru de l'auteur, qui doit penser à Virgile (Anchise aux Enfers) et à l'Arioste (Merlin), soit autant de récits anticipés de l'histoire ultérieure, sous forme de prophéties. Reprend pourtant ensuite la distinction fabula historia argumentum, comme "matiere de la narration"; distingue des "parties » qui sont des lieux, plus ou moins canoniques; six sortes de "disposition": "directe ou historique", « indirecte ou romanesque " (c'est l'ordo artificialis, l'ordre artificiel) et trois autres sortes, qui semblent dériver de Ramus; uirtutes: succinctes, claires, probables, excitantes. Ce dernier adjectif est lié par Bary à « la rigueur ou la pitié ». Il pourrait donc correspondre à la suauitas, comprise au sens du dulcia de l'Art poétique d'Horace (v. 99), «dulcia sunto »: les poèmes doivent être non pas «doux » mais «pathétiques». Suivent des déterminations inhabituelles pour «l'excitation»: amplification, exclamations, etc.

73 Jacques Hautin (1599-1671), Rhetorica... (1647), Munich, Lukas Straub, 1657, II « De dispositione », chap. 6 « De narratione », p. 105-115

Jésuite. Reprend Part., avec ses quatre uirtutes, en contaminant avec d'autres textes, commente rapidement et donne des exemples.

74 Jacques Du Roure (mort en 1670), Rhetorique françoise, Paris, Chez l'Auteur, 1662, p. 44

Diverses espèces : fabuleuses / veritables; continue / interrompue; insiste sur l'art de dissimuler; exemples développés; selon l'ordre du temps, des choses ou des actions; quatre «qualités»: Clarté, Vray-semblance, Agrément, Breveté ; déterminations (Agrément: "choses extraordinaires ou dites d'une façon extraordinaire ", sans doute reprise des Part. 22 et 31), exemples (Héliodore, les Éthiopiques / Voiture). predicateurs, Paris, Chez l'Autheur, 1665

Élimine la narration dans la disposition.

Le Gras (prénom et dates inconnus), La Rethorique Françoise ou les Preceptes de l'ancienne et vraye eloquence, Paris, Antoine de Raffle, 1671, chap. 3 « De la Narration », p. 111-129.

Suit Inv. et Her., pour l'essentiel. Trois «sortes»; personnes et choses; fable (Lucien), histoire et argument (Énéide); différences selon les genres oratoires; exemples développés; vertus (pas de terme général utilisé) : Clarté, Breveté, VraySemblance; développements usuels; insiste sur le caractère "moral» (morata, repris d'Aristote); consignes relatives à la validité de la cause (usuel); à la conduite de la narration; ajoute une section «Du Stile propre à la Narration» (p. 127) : reprend les consignes relatives à la suavitas de Part., en soulignant qu'elle «doit avoir un air agreable, des surprises qui donnent de l'admiration ", " estre remplie de douceur et de gravité »; mais fusionne avec la festiuitas d'Inv. et Her., en donnant la liste des procédés des deux ensembles de traités, amalgamés. Enchaîne sur la digression (p. 130).

Bernard Lamy (1640-1715), La Rhétorique ou l'Art de parler (1672), Paris, André Pralard, 1688, V, 18, p. 355-358 ; éd. Ch. Noille-Clauzade, Paris, Honoré Champion, 1998

Oratorien. Dans le titre du chapitre, ne parle pas de narration, mais de "proposition»; peut être succincte ou consister en un « récit» circonstancié et une " peinture qui l'expose aux yeux des juges »; sincérité et simplicité; choix des circonstances; uirtutes : courte, claire, probable; déterminations usuelles rapides. Sommaire.

Étienne Dubois de Bretteville (1650-1688), L'Éloquence de la chaire, et du barreau (1689), Paris, Denis Thierry, 1698 
Au livre II, chap. 3 « De la Proposition, de la Division, et de la Narration, par rapport au Barreau », p. 132 sq. : «proposition » (au sens de "simple exposition du fait »); narration (p. 137-146) : sommaire ; « la plus courte que l'on pourra », employer « les plus vives et les plus sublimes Figures de l'Eloquence, pour donner un tour extraordinaire aux faits que l'on raconte »; exemples (Le Maître, Patru). Le chapitre suivant (II, 4) s'intitule « De la Proposition, de la Division, et de la Narration par rapport à la Chaire », p. 146 : en fait, ne traite que de la division... 3, 103) », Les Noms du style dans l'Antiquité gréco-latine, textes présentés et édités par Pierre Chiron et Carlos Lévy, Peeters, Louvain, 2010, p. 113-129. mutations de la théorie du style à l'époque hellénistique, Paris, Vrin, 2001, p. 117-172 et 302-304.

Sa deuxième partie, «De Dispositione oratoria », énumère les parties du discours, mais passe directement de l'exorde (chap. 1) à la confirmatio (chap. 2). L'absence ici de la narratio tient probablement au fait que Jouvancy renvoie la narration aux progymnasmata, qu'il traite à la fin de son volume, en se fondant sur Aphtonius (voir supra).

\section{Bibliographie}

Marie Formarier, "La narratio chez Cicéron doit-elle être brève pour persuader ? ", Interférences (http://journals.openedition.org/interferences/6007), $\mathrm{n}^{\circ}$ 10, 2018.

3 Paula Olmos Gómez, «La preceptiva sobre la narratio en los rétores latinos », Revista de estudios sociales, 44, déc. 2012, p. 62-74 (Open Éditions). Fournit des indications intéressantes (commentaire de Marius Victorinus sur le Inv., Agricola, Zabarella - qui nie la valeur de la narration -, divers auteurs des Rhetores latini minores) ; à compléter, cependant (Her., Part., Progymnasmata, notamment, et notre inventaire, pour l'humanisme).

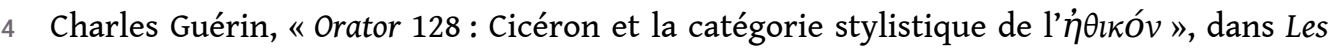
Noms du style..., op. cit., p. 143-164.

Véronique Montagne, « Le De suaui dicendi forma de Jean Sturm : notes sur la douceur du style à la Renaissance ", Bibliothèque d'Humanisme et Renaissance, tome LXXVI, 2004, $\mathrm{n}^{\circ} 3$, p. 541-563.

\section{Ordo naturalis / ordo artificialis (ordre naturel / artificiel)}

Traité en détail par Edmond Faral, Les arts poétiques du XII et du XIII siècle, Paris, Champion, 1925, p. 55-59. Cite Her. (III, « $9 »$, en fait, 16), Fortunatien, Sulpitius Victor, Martianus Capella, Horace (Art poétique, v. 42-45), le pseudo-Acron, les Scholia uindobonensia ad Horatii artem poeticam, le commentaire de Ti. Donat sur Virgile, et toute une série de médiévaux, dont les auteurs qu'il édite dans son recueil.

Motif repris dans les commentaires renaissants d'Horace, ad loc.: voir le site Renaissances d'Horace http://ihrim.huma-num.fr/nmh/Horatius/) de Nathalie Dauvois et al.

On notera que cette distinction opère sur un champ plus large que celui, devenu 
classique, du récit poétique. Il concerne aussi l'ordre d'exposition oratoire, même lorsqu'il ne s'agit pas d'une narration à proprement parler.

C'est le point de vue de Her. III, 16, qui distingue deux genera dispositionum ou "genres de disposition »: " unum ab institutione artis profectum, alterum ad casum temporis adcommodatum», "l'un dicté par les préceptes techniques, l'autre déterminé en fonction des circonstances ». Cette distinction ne recouvre pas encore tout à fait la distinction canonique entre "artificiel » et " naturel », ni, surtout, celle de Fortunatien (Ars Rhetorica, III, Halm, Rhetores latini minores, op. cit., p. 120, cité par Faral, op.cit., p. 55). Fortunatien distingue en effet huit formes d'ordo artificialis, doctrine devenue classique au Moyen Âge.

Théon, Progymnasmata, op. cit.

Il s'agit du passage, mentionné supra, sur l'oeconomia ou organisation narrative de l'Odyssée (p. 278 de l'éd. 1541 ; Patillon p. 48-49 = Spengel 86). En voici le début (trad. Patillon) : «Le bouleversement de l'ordre se fera de cinq façons. Nous pouvons en effet commencer au milieu [apo tôn mesôn], remonter de là au début, puis aller jusqu'à la fin, comme l'a fait Homère dans l'Odyssée : il a commencé à l'époque du séjour d'Ulysse chez Calypso, après quoi il est remonté au début, non sans quelque raffinement dans la démarche [oikonomias glaphuras], puisqu'il a imaginé Ulysse faisant aux Phéaciens le récit de ses propres aventures; puis il a enchaîné la suite du récit, qu'il a conduit jusqu'à son terme, jusqu'à ce qu'Ulysse ait tué les prétendants et se soit réconcilié avec leurs parents. »

Latini, Li livres dou Tresor, op. cit., p. 482-485

Très développé. Extension très large. Distingue les huit formes d'ordre artificiel, comme Fortunatien.

Agricola, De inuentione dialectica, op. cit.

$\mathrm{Au}$ livre III, revient longuement et à plusieurs reprises sur la notion. Chap. 6 «Quae sit ad breuiter dicendum via » (p. 405-407, Mundt, p. 472-478), «Sur la méthode pour être bref » : la réflexion s'appuie sur l'exemple de l'Énéide et de l'Andrienne. Chap. 8, pose une tripartition, ordo naturalis, arbitrarius, artificialis, "ordre naturel", «libre », «artificiel» (p. 413-414, Mundt, 1.35-55, Poel p. 250-253) : le premier est l'ordre que suivent les annales, une année suit l'autre ; le second renvoie à la liberté de l'historien (exemple de Tite-Live); le troisième est exemplifié par le début de l'Énéide (le récit de la tempête récente précède celui de la chute de Troie). Chap. 9 "Quis poetis ordo, quis historiae, quis tradendis artibus conueniat", "Quel ordre convient pour la poésie, pour l'histoire et pour l'enseignement » : assez similaire au précédent, et détaillé ; exemples de l'Énéide et de l'Andrienne, à nouveau, de façon là aussi très détaillée.

9 Ramus (Pierre de La Ramée, 1515-1572), Dialectique de Pierre de la Ramée, II, Paris, André Wechel, 1555, p. 229-231

Cite Horace, Art poétique, v. 42-45, longuement; donne l'exemple canonique de l'Énéide et celui de l'Eunuque de Térence, avec une longue analyse de cette pièce (sans doute fortement inspirée de Donat et des commentateurs ultérieurs) ; signale que les poètes comiques font « que toutes choses y semblent estre fortuites et inopinées » (p. 230), ce qui provient d'Inv.-Her. et Part.

Amyot, L'histoire aethiopique de Heliodorus..., Paris, Jean Longis, 1547, préface, n. p. (vue 10 de l'exemplaire numérisé par la BnF, IFN-8600170, sur Gallica)

Reprend Strabon et Donat (sans le dire, pour le second) : «Pour autant que l'artifice d'inuention Poëtique, comme doctement à escrit Strabon, consiste en trois choses ", etc. On remarquera que l'ordo artificialis est en revanche refusé par Amyot pour l'éloquence royale (voir supra). 
Scaliger (Giulio Caesare Scaligero, 1484-1558), Poetices libri VII, Lyon, Antoine Vincent, 1561, III, 96, p. 144 ; Sieben Bücher über die Dichtkunst, éd. et trad. allemande de Luc Deitz, Stuttgart/Bad Cannstatt, Friedrich Frommann, t. III, 1995, p. 20-25

Si le livre III («Idea») n'est pas traduit en français, on trouvera une traduction partielle du chapitre 96 (la seule colonne de gauche de la p.144) dans Théories poétiques néo-latines, dir. Virginie Leroux et Émilie Séris, Genève, Droz, 2017, p.732-734. Ne recourt pas à la terminologie canonique, mais développement détaillé. La narration épique ne doit pas commencer $a b$ ouo : "Comme le prescrit Horace [Art poétique, v. 147], il ne faut surtout pas remonter à l'œuf. Que ce soit notre premier précepte : il faut commencer par un événement fameux et enchaîner avec des faits apparentés et très proches" (p.732, avec exemple de Lucain). "Second précepte: il ne faut pas mener le récit en ligne droite de peur de faire naître l'ennui », afin que «l'esprit de l'auditeur reste en suspens car il attend ce qui n'est pas encore exposé. L'unique ou la principale exigence est la suivante : captiver l'auditeur [auditorem quasi captiuum detinere] » (p. 732-734, avec exemple de l'Énéide puis des Éthiopiques, dont il trouve la disposition «splendide»). Sur le second précepte, voir Donat et Terence Cave, infra. 
Repris d'Evanthius : sur les personnages protatiques (p. 19); sur les quatre parties de la comédie (p. 22); De comoedia: autre présentation des quatre parties, avec précisions sur la protasis, qui n'expose qu'une partie de l'argument et diffère le reste, pour tenir en suspens le spectateur (p. 27) ; analyse de l'Andrienne de Térence (Andria, I, 1-2), p. 35 : souligne la présentation de la narratio en acte, qui n'est pas ennuyeuse, bien que longue. Propose la théorie classique de l'exposition. Absolument essentiel. L'ensemble du commentaire est de toute façon d'une importance capitale, notamment du point de vue de la narratio.

Dans sa préface ou praefatio à l'Eunuque de Térence (Eunuchus), p. 265-266: désignation des parties de la comédie; théorie explicite du suspens narratif (la première de l'histoire de la rhétorique, à mon sens, trop négligée - y compris par

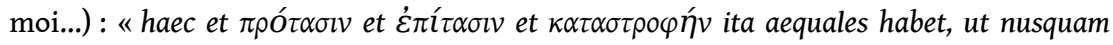
dicas longitudine operis Terentium delassatum dormitasse. Actus sane implicatiores sunt in ea et qui non facile a parum doctis distingui possint, ideo quia tenendi spectatoris causa uult poeta noster omnes quinque actus uelut unum fieri, ne respiret quodammodo atque, distincta alicubi continuatione succedentium rerum, ante aulaea sublata fastidiosus spectator exsurgat. » « La pièce montre dans la protase, l'épitase et la catastrophe une si grande égalité que l'on pourrait dire que nulle part Térence, malgré la longueur de l'œuvre, n'a éprouvé de fatigue ou s'est endormi. Les actes y sont particulièrement imbriqués et difficiles à séparer si l'on n'est pas tant soit peu spécialiste, parce que, pour tenir en haleine le spectateur, notre poète veut que les cinq actes n'en fassent pour ainsi dire qu'un, afin qu'il n'ait pas le temps de respirer et que, devant un enchaînement d'événements qui se succèdent avec de rares pauses ici ou là, le spectateur fatigué ne quitte pas le théâtre avant le lever de rideau » (texte et trad. du site HyperDonat ; " avant le lever de rideau » signifiait « avant la fin de la pièce », car à Rome, la pièce commence quand le rideau tombe, et finit quand il se lève). Etc. Cremonensi. Iodoco Badio Ascensio, Paris, Josse Bade, 1514

Gellio Bernardino Marmitta (1440 ?-1497 ?), Daniele Gaetano (Gaietanus ou Cajetan, etc., mort en 1528) et Josse Bade (1462-1535) : ces trois commentateurs humanistes s'inspirent fortement du commentaire de Donat à Térence (qu'ils citent) et appliquent aux tragédies de Sénèque ses analyses sur les scènes d'exposition. Une des sources probables de Dubois sur ce point.

En fait de commentaires humanistes, voir aussi ceux d'Horace (Landino, Bade, Denores, Achilles Statius, Lambin, etc.), sur le site de N. Dauvois et al., http:// ihrim.huma-num.fr/nmh/Horatius/.

Dubois, Poetica, op. cit., $\mathrm{f}^{\circ}$ aiiii $\mathrm{r}^{\mathrm{o}}$

Faire parler un personnage en proie à une émotion vive (provient de Donat et des commentaires de Sénèque cités à l'instant).

Ramus, Dialectique, op. cit.

Voir supra.

Carbone, De causis..., op. cit., III, p. 364-366

Principe repris d'Horace. Analyses de Virgile. Sa septième thèse (ou " positio ») explicite la place de l'inuocatio : l'ordre «naturel » à suivre par le poète épique est d'abord la propositio ou sujet de l'œuvre, puis l'inuocatio ou invocation (à la Muse, etc.), enfin la narratio (" prius proponere, postea inuocare, \& postremo loco narrare", p. 365) - ce qui vient de Servius, Commentaire à l'Énéide (sur Aen. I, 8) : «In tres partes Poetae diuidunt carmen suum, proponunt, inuocant, narrant ", " les poètes divisent leur poème en trois parties: ils proposent, invoquent, racontent» (cité et traduit dans V. Leroux et É. Séris, Théories poétiques, op. cit., p. 722). 


\section{Bibliographie}

Marchetti, Paris, Belles Lettres, t. I, 2003, p. 6-9

Le «songe de Scipion » est le célèbre passage de la République de Cicéron (VI, 9-29), qui a été conservé précisément par le néoplatonicien Macrobe. Celui-ci médite sur le recours des philosophes, et notamment Platon, à la fiction (fabula, fabulosa ; incipit du passage : "Nec omnibus fabulis philosophia repugnat », "La philosophie ne répugne pas toujours aux fictions", trad. Armisen-Marchetti, ici et ensuite). La formule même de narratio fabulosa est au § 9 : parmi les «fables", dans certaines "l'argument relève de l'imagination» («argumentum ex ficto»), c'est le cas des fables d'Ésope ; « dans d'autres en revanche l'argument s'appuie bien sur une base véridique solide, mais cette vérité même est présentée à travers un agencement imaginaire, et on parle alors de narration fictive, non de fiction [narratio fabulosa, non fabula] : ainsi les rites des mystères, les récits hésiodiques ou orphiques au sujet de la généalogie et des aventures des dieux, les formules mystiques des Pythagoriciens. »Cette seconde catégorie est à son tour divisée en deux (§11): le philosophe ne peut cautionner que la seconde sous-espèce, celle où «la connaissance du sacré est révélée sous le voile pieux d'éléments imaginaires [sub pio figmentorum uelamine], couverte de faits honnêtes et revêtue de noms honnêtes ». Essentiel pour tout le Moyen Âge, notamment, Boccace, etc.: principale source, médiée par Isidore de Séville, et avec Aristote, Métaphysique (commentée par Saint Thomas, d'après Simplicius) de l'idée de l'integumentum poeticum, ou «'cryptage' poétique ». En distinguant entre les fictions qui conviennent aux philosophes et les autres, Macrobe ne se contente pas de proposer une taxinomie des fictions: au passage, il fournit une description d'ensemble du champ de la fiction. On notera que, chez Cicéron, le Songe de Scipion, comme le récit d'Er dans la République de Platon (livre $\mathrm{X}, 614 \mathrm{~b}-621 \mathrm{~d}$ ), est lui-même un récit inséré dans le dialogue, à des fins démonstratives.

Érasme, De duplici copia, II, « De exemplo fabuloso », op. cit., éd. 1703, t. 1, p. 90-92 ; éd. 1988 , p. $234-240$

Melanchthon, De Rhetorica libri tres, I, op. cit., p. 29-41

Sur l'interprétation allégorique des fabulae ou fictions ; important pour ce thème.

Caesarius, Rhetorica, III, chap. 14, op. cit., p. 55-56

Sur les fables des poètes comme arguments. Proche d'Érasme.

Voir aussi Lorichius, commentaire d'Aphthonius, op.cit. Et, en général, tous les Progymnasmata, au chapitre Fabula : notamment Jouvancy, L'Élève de rhétorique, V, 2 « De narratione fabulosa », op. cit., p. 264-285. 


\section{Bibliographie}

110 Marie-Madeleine Fontaine, introduction à B. Aneau, Alector, op. cit., p. XL-LXVIII.

111 Stéphanie Lecompte, La chaîne d'or des poètes. Présence de Macrobe dans l'Europe humaniste, Genève, Droz, 2009 (sur la théorie de la fable chez Macrobe et sa descendance au XVI ${ }^{\mathrm{e}}$ siècle).

112 Charles Méla, «Poetria Nova et Homo novus ", Littérature, n 74, 1989, p. 4-26 (sur la théorie de la narration, et en particulier de l'ordo, au Moyen Âge).

\section{L'histoire}

Lucien, Comment il faut écrire l'histoire,trad. Émile Chambry, dans Lucien, Euvres complètes, Paris, Garnier, t. II, 1933, p. 1-30

Fondamental. Les œuvres complètes de Lucien ont été disponibles en traduction latine à partir de 1538 (Opera omnia, Francfort, Christian Egenolff), mais nombre de ses opuscules ont été traduits auparavant.

\section{ANNEXES}

\section{Annexe 1 : Quelques commentaires des Divisions de l'art oratoire}

Giorgio Valla (1447-1500), Commentationes. In Ptolomei quadripartitu[m] inq[ue] Ciceronis Partitiones [et] Tusculanas questio[n]es..., Venise, Simone Bevilacqua, 1502

Marque le retour d'intérêt pour ce traité de Cicéron; mais assez succinct. Ne

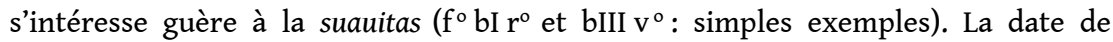
rédaction est incertaine. Le commentaire est repris dans l'ouvrage suivant, de D’Estrebay. 
Jacques-Louis d'Estrebay (Strébée ou Strebaeus, mort vers 1550), M. Tvl. Ciceronis, De partitione oratoria, dialogus (1533), Cologne, Johann Gymnich (Gymnicus), 1539,

p. $116-122$

Détaillé, avec analyse d'un exemple cicéronien, Actio III in Verrem; s'intéresse de plus près à la suauitas que G. Valla. Renvoie à Quintilien, mais des traces vraisemblables aussi d'Agricola; insiste sur le suspens ("non sine exspectatione iucunda », «non sans une agréable expectative ») dans son analyse de la suauitas de la narration de Cicéron, et y identifie les divers procédés de la liste. - La date de la princeps est celle que donne Renaissance rhetoric, op. cit., p. 111 (leur référence RR 864).

Johannes Sturm (1507-1589), In partitiones oratorias Ciceronis dialogi quatuor (1545), III, Strasbourg, Blasius Fabricius, 1549 (colophon de 1554), p. 166-170

Détaille les Divisions de l'art oratoire, avec des indications sur les $\pi \varepsilon \rho l \pi \varepsilon ́ \tau \varepsilon l \alpha l$ tragicae ("péripéties tragiques», p. 168, sur Part. 31-32), etc., pour la suauitas (recours à Aristote, Poétique). Utilise souvent Hermogène et assimile les uirtutes aux ideai de celui-ci. - Peter Mack (A history..., op. cit., p. 133) précise l'historique de l'ouvrage : en 1539, le commentaire de Sturm n'a que deux livres ou « dialogues » (même titre, avec «dialogi duo ») ; il en a quatre à partir de 1545. Ajoutons que les deux premiers livres s'arrêtent à Part. 25 : c'est donc au commentaire complet que nous nous référons.

Marcantonio Majoragio (Majoragius, 1514-1555), M. Antonii Maioragii, commentarius in dialogum de Partitione Oratoria, Venise, Francesco de' Franceschi, 1587, fo $83 \mathrm{v}^{\circ}-87 \mathrm{r}^{\mathrm{o}}$

Composé avant 1555, éditions posthumes au moins à partir de 1569 (Milan, Pacificus Pontius). Détaille fortement les quatre uirtutes proposées par le traité cicéronien (voir supra), avec séries de rubriques (15 pour la probabilitas, 10 pour la suauitas). Donne un véritable petit traité de la narration, et en particulier de la narration captivante. Sera largement recopié par Caussin (voir S. Duval et L. Charles dans le présent numéro). Renvoie souvent à Valla et Agricola.

Il va de soi que nous nous limitons ici aux commentateurs les plus importants, et les plus significatifs pour notre sujet, la narratio : lorsque Peter Mack restitue l'immense succès éditorial des Divisions à l'époque, il souligne ainsi que « les commentaires de Giorgio Valla, Strebaeus, Latomus et Sturm semblent avoir joué un rôle important dans cette diffusion » (A history..., op. cit., p. 20, nous traduisons). Pour mémoire, voici, par ordre alphabétique, la liste des commentateurs des Divisions que cite Renaissance rhetoric (p. 119-122) : Gerardus Bucoldianus, Joachim Camerarius, Léger du Chesne (Leodegarius a Quercu), Johannes de Fossa, Christoph Hegendorph, Denys Lambin, Bartholomaeus Latomus, Marcantonio Majoragio, Philipp Melanchthon, Claude Mignault, Jacques-Louis Strébée, Johannes Sturm, Giorgio Valla. Et scholies, notes ou résumés de : Veit Amerbach, Celio Secundo Curione, Valentinus Erythraeus, Jean Mercier, Guillaume Morel, Omer Talon, Adrien Turnèbe.

\section{Annexe 2 : Liste exploratoire d'éditions commentées du Pour Milon, avec (ou sans !) étude de la narratio aux $\mathrm{XVI}^{\mathrm{e}}$ - $\mathrm{XVII}{ }^{\mathrm{e}}$ siècles}

On pourrait évidemment envisager un répertoire complet des analyses de discours, cicéroniens ou autres, s'intéressant à la narratio. Nous retenons celles du Pour Milon à cause du caractère exemplaire prêté à ce discours, en matière de 
narration, par la plupart des humanistes. Liste non limitative. Pour les seuls discours de Cicéron, une longue liste de commentateurs est donnée dans Renaissance rhetoric (op. cit., p. 127-128), suivie de la liste des éditions (p. 128-135).

Agricola, De inuentione dialectica, op. cit.

Au chapitre 4 du livre II (p. 200-201, Mundt 1. 81-125), analyse de type dialectique, par lieux et syllogismes; mais insiste sur la delectatio, inséparable de l'argumentation (voir supra); image de la nourriture et du plaisir du goût (II, 5, p. 204-205, Mundt p. 224-229). La liste des passages où Agricola cite le Pour Milon est donnée dans l'index de l'éd. Mundt, op. cit., p. 745.

Dubois, F. Sylvii [...] pro Milone commentarii, op. cit.

Voir supra. Dubois a commenté de nombreux autres discours de Cicéron: voir la liste complète dans Renaissance rhetoric, op. cit., p. 173-174.

Claude Baduel (1491-1561), Claudii Baduelli annotationes in M. T. Ciceronis pro Milone et pro M. Marcello orationes (quarum textus inest) quibus adjectae sunt ejusdem orationes aliquot ab ejus discipulis in gymnasio Nemausensi pronuntiatae..., Lyon, Sébastien Gryphius, 1552

Très intéressant et détaillé.

In omnes M. Tullii Ciceronis orationes..., Bâle, Robert Winter, 1539

Compilation composée après la découverte des commentaires de Dubois par l'éditeur scientifique, Jacobus Bedrotus (Jacob Bedrott, 1493/97-1541). Pour Milon: col. 851 (Dubois) ; col. 922 (Melanchthon, simple canevas); col. 929 (Latomus: narratio, col. 936 ; surtout dialectique, référence à Agricola). - Dans Renaissance rhetoric, la notice (op.cit., p.130, RR 1066) donne la liste complète des commentateurs réunis par cet ouvrage.

Cavalcanti, Della retorica , op. cit., p. 442

Denys Lambin (1520/21-1572), Orationum M. T. Ciceronis..., Strasbourg, Josia Rihel et Jacques Dupuis, 1581, vol. I, p. 113 sq.

Simples rubriques marginales.

Orazio Toscanella (1520 ?-1579 ?), Oratio Ciceronis pro T. Annio Milone ab Horatio Tuscanella examinata, Venise, Petrus de Francisciis, 1576

Marcus Beumler (Bäumler, 1555-1611), M. T. Ciceronis oratio pro T. Annio Milone, Analysis Logica, Rhetorica, Ethica, Politica, Historica, à Marco Beumlero Tigurino illustrata, Spire, Bernhard Albin (Albinus), 1586, p. 50-59

S'intéresse principalement à la présentation des causes et des circonstances.

Pelletier, Reginae Palatium, op. cit.

Voir supra.

Jouvancy, L'Élève de rhétorique, op. cit., VI « Synopsis orationum », chap. 3, art. 2, p. 444-447

Dans son résumé ou synopsis du discours, ne parle pas de la narratio du Pour Milon, mais décrit seulement deux parties de la confirmatio.

\section{NOTES}

1. Lawrence D. Green et James J. Murphy, Renaissance rhetoric. Short-title catalogue 1460-1700, Burlington, Ashgate, 2006.

2. $\mathrm{NdE}$-Le récit " politique » est « celui que les orateurs emploient dans les procès », comme le précise Aphthonios, cité, dans ce même numéro, par Lise Charles et Suzanne Duval («La 
narration, par Majoragius... », note 61). La cité se disant en grec polis, l'adjectif politikos peut signifier " civil, public, qui relève de la cité (citadin, citoyen)» (Germaine Aujac, «Lexique général ", s. v. politikos, dans son édition de Denys d'Halicarnasse, Opuscules rhétoriques, Paris, Belles Lettres, t. V, 1992, p. 263). Le mot a donc pour strict équivalent latin l'adjectif ciuilis.

\section{AUTEUR}

\section{JEAN LECOINTE}

Poitiers Forellis B 2 